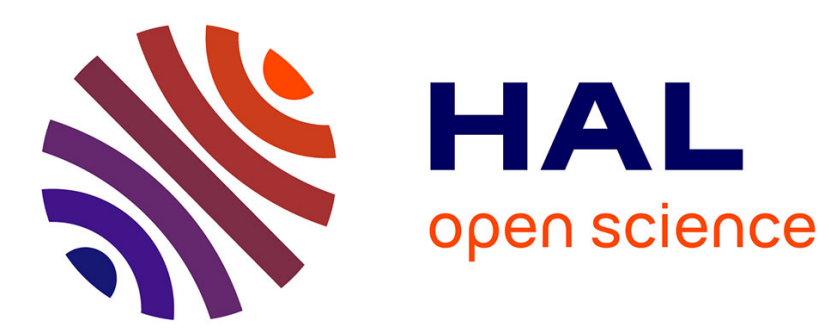

\title{
L'estimation du rayonnement solaire au sol par la nouvelle méthode Heliosat-4
}

Philippe Blanc, Lucien Wald

\section{To cite this version:}

Philippe Blanc, Lucien Wald. L'estimation du rayonnement solaire au sol par la nouvelle méthode Heliosat-4. La Météorologie, 2015, 90, pp.53-61. hal-01180387

\section{HAL Id: hal-01180387 \\ https://hal-mines-paristech.archives-ouvertes.fr/hal-01180387}

Submitted on 26 Jul 2015

HAL is a multi-disciplinary open access archive for the deposit and dissemination of scientific research documents, whether they are published or not. The documents may come from teaching and research institutions in France or abroad, or from public or private research centers.
L'archive ouverte pluridisciplinaire HAL, est destinée au dépôt et à la diffusion de documents scientifiques de niveau recherche, publiés ou non, émanant des établissements d'enseignement et de recherche français ou étrangers, des laboratoires publics ou privés. 


\section{Résumé}

Les projets MACC, MACC-II et MACC-III (Monitoring atmospheric composition and climate), financés par la Commission européenne, fournissent des données décrivant la composition de l'atmosphère en gaz et aérosols. Une nouvelle méthode, Heliosat-4, a été conçue et réalisée à Mines ParisTech afin de produire des estimations de l'irradiation solaire au sol, utilisée notamment par les producteurs d'électricité pour le dimensionnement et la surveillance des centrales solaires. Les estimations du rayonnement solaire global, direct et diffus, sont de qualité similaire ou meilleure que les autres méthodes existantes. Un autre résultat des projets MACC est le modèle de ciel clair McClear qui se révèle être d'une grande précision.

\section{Abstract}

The estimation of solar radiation at ground level by the new Heliosat-4 method

The MACC, MACC-II and MACCIII (Monitoring atmospheric composition and climate) projects, funded by the European Commission, provide data on atmospheric composition for both gases and aerosols. A new method Heliosat- 4 has been designed to provide estimates of the solar radiation at ground level. These estimates are used for the sizing and monitoring of solar plants for electricity production. Estimates of the direct, diffuse and global radiation by Heliosat-4 are of better or similar quality to those given by current methods. Another result of MACC is the McClear clear-sky model that proves to be of high accuracy.

\section{L'estimation du rayonnement solaire au sol par la nouvelle méthode Heliosat-4}

\author{
Philippe Blanc, Lucien Wald \\ Centre observation, impacts, énergie, Mines ParisTech, PSL Research University, \\ Sophia Antipolis \\ lucien.wald@mines-paristech.fr
}

'énergie d'origine solaire arrivant sur la Terre est la principale source d'énergie externe. Elle est évidemment une variable très importante pour le climat, la météorologie ou encore la photosynthèse. Elle est également une source de production d'électricité via des systèmes de conversion comme les panneaux photovoltaïques (PV) ou des systèmes thermodynamiques à concentration (figure 1). C'est cet usage de l'énergie solaire qui guide les travaux présentés ici.
De nombreux pays favorisent l'installation de centrales solaires, souvent pour accroître leur indépendance énergétique vis-à-vis d'autres pays et d'autres sources de production d'électricité comme les énergies fossiles et nucléaires, mais aussi pour diminuer leurs émissions de gaz à effet de serre. La décision de réalisation d'une centrale en un lieu précis, sa conception, son installation et le suivi de son fonctionnement requièrent la connaissance du rayonnement solaire incident au sol.

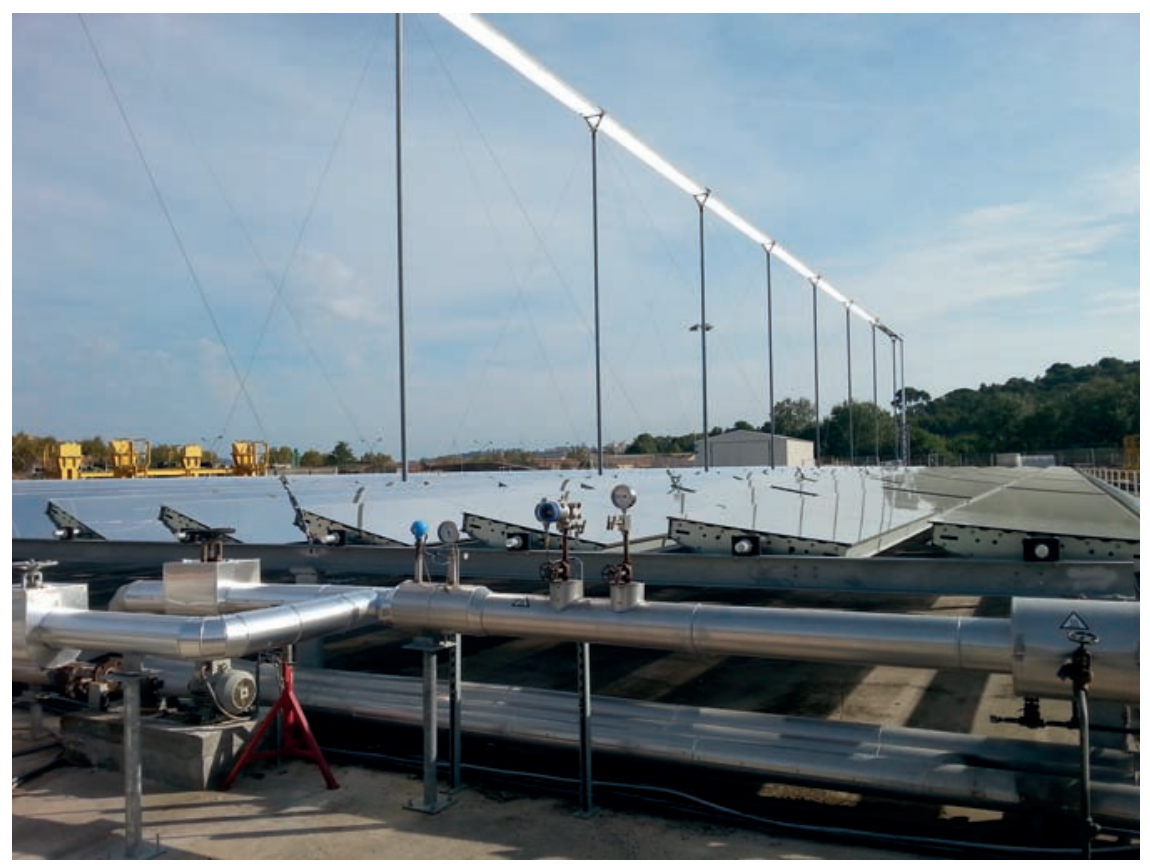

Figure 1. Prototype d'une installation de conversion thermique du rayonnement solaire sur le site de la société CNIM à La-Seyne-sur-Mer dans le Var. Le rayonnement provenant directement du Soleil est concentré par des réflecteurs linéaires de Fresnel sur un tube, en haut sur la photo, dans lequel circule un fluide caloporteur. Le fluide produira de la vapeur qu'une turbine convertira en électricité (crédit photo : Roseline Adde-Wald). 
Les projets MACC, MACC-II et MACC-III (Monitoring atmospheric composition and climate), financés par la Commission européenne, ont pour objectif la mise en place d'un dispositif opérationnel décrivant la composition de l'atmosphère en gaz et aérosols qui permet à la fois d'effectuer des réanalyses depuis 2004 et des prévisions pour les trois jours à venir. Les projets MACC comportent également comme objectif secondaire la fourniture d'estimations du rayonnement solaire incident au sol, visant à satisfaire principalement les besoins des installateurs et opérateurs de centrales solaires. Cet article présente la manière dont ces estimations sont faites et indique les principaux apports des projets MACC par rapport aux méthodes d'estimation existantes.

\section{Le rayonnement solaire au sommet de l'atmosphère}

Le Soleil émet de manière isotrope un rayonnement important dû à sa température superficielle élevée. La Terre reçoit une partie de ce rayonnement. Le flux énergétique reçu par la Terre est appelé éclairement énergétique*, abrégé en éclairement. La moyenne annuelle de cet éclairement reçu sur un

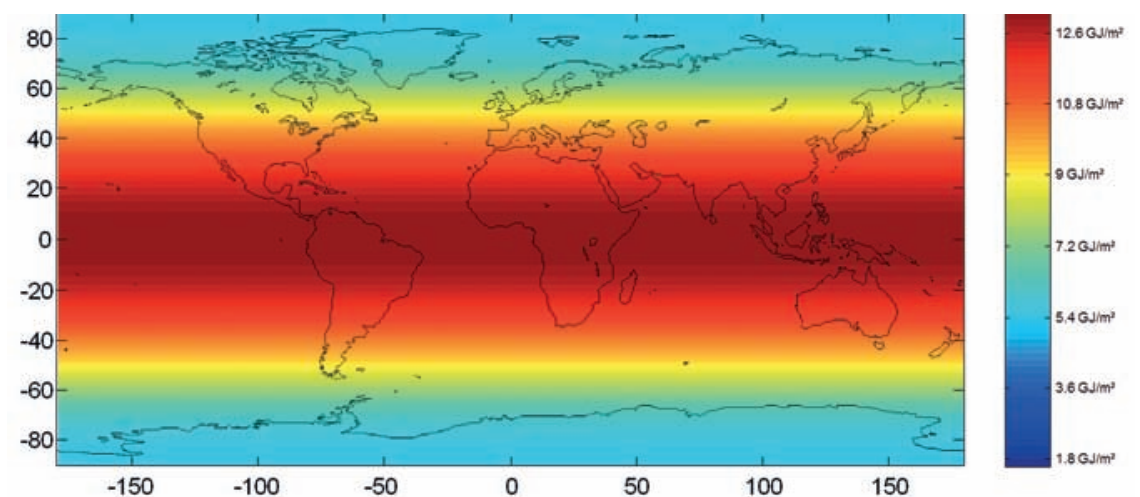

Figure 2. Irradiation annuelle au sommet de l'atmosphère, c'est-à-dire l'énergie reçue durant une année par un plan horizontal situé au sommet de l'atmosphère, exprimée ici en GJ m-2. Les continents ont été tracés pour servir de repère. On peut observer que cette irradiation ne dépend que de la latitude (axe vertical). Les maxima, en rouge sombre, sont situés dans la zone équatoriale, les minima, en bleu, dans les zones polaires.

plan normal aux rayons du Soleil situé au sommet de l'atmosphère est appelée constante solaire* ; sa dernière estimation est de $1362 \mathrm{~W} \mathrm{~m}^{-2}$ (Meftah et al., 2014).

L'éclairement dépend de la distance entre notre planète et le Soleil, laquelle varie selon les saisons. Plus cette distance est grande, plus l'éclairement est faible. Ainsi l'éclairement reçu au cours de l'année par un plan normal aux rayons du Soleil situé au sommet de l'atmosphère varie-t-il entre 0,97 et 1,03 fois la constante solaire. L'activité solaire, manifestée

\section{Définitions}

L'angle solaire zénithal $\theta_{\mathrm{S}}$ est l'angle formé par la verticale du lieu considéré et la direction du Soleil.

L'éclairement énergétique est le flux énergétique solaire reçu par unité de surface. I s'exprime en $\mathrm{W} \mathrm{m}^{-2}$.

L'irradiation est l'intégrale de l'éclairement durant un certain temps. C'est cette quantité qui est mesurée dans les réseaux météorologiques. Elle est exprimée en

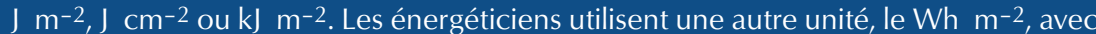

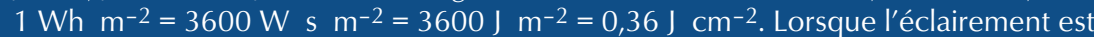
déduit d'une mesure, on devrait mentionner la période d'intégration. Par exemple, on devrait parler de la moyenne horaire de l'éclairement si celui-ci est déduit d'une mesure d'irradiation horaire. Cette précision est souvent omise s'il n'y a pas de risque de confusion.

L'éclairement au sommet de l'atmosphère sur un plan normal aux rayons du Soleil $E_{0 n}{ }^{t}$ varie avec le temps $t$ avec un cycle saisonnier principalement. Sa moyenne sur une année est appelée constante solaire $E_{0 n}$, avec $E_{0 n}=1362 \mathrm{~W} \mathrm{~m}^{-2}$ et $E_{0 n}{ }^{t}=E_{0 n} \pm 3 \%$.

L'éclairement au sommet de l'atmosphère sur un plan horizontal $E_{0}{ }^{t}$ est donné par $E_{0}{ }^{t}=E_{0 n}{ }^{t} \cos \left(\theta_{\mathrm{S}}{ }^{t}\right)$.

L'éclairement reçu au sol sur un plan horizontal est appelé éclairement global G. II est la somme de la composante directe B reçue de la direction du Soleil et de la composante diffuse $D$ provenant de toutes les autres directions de la voûte céleste, avec $G=B+D$.

L'éclairement au sommet de l'atmosphère ou au sol a une distribution spectrale non uniforme, c'est-à-dire qu'il dépend de la longueur d'onde. On appelle éclairement total l'intégrale de l'éclairement sur le spectre.

par les taches solaires, modifie très légèrement cet éclairement d'environ $0,1 \%$, avec un cycle de 11 ans sur lequel se superposent parfois des variations beaucoup plus rapides d'un jour sur l'autre ou d'une semaine sur l'autre, dont l'amplitude peut atteindre $5 \mathrm{~W} \mathrm{~m}^{-2}$, soit environ $0,3 \%$ de la constante solaire (Kopp et Lean, 2011).

L'angle solaire zénithal* $\theta_{\mathrm{S}}$ joue un rôle majeur sur l'éclairement reçu sur un plan horizontal au sommet de l'atmosphère. Plus cet angle est élevé, plus le Soleil est proche de l'horizon et plus cet éclairement est faible. En effet, l'éclairement sur un plan horizontal $E_{0}{ }^{t}$ est égal au produit de l'éclairement sur plan normal $E_{0 n}{ }^{t}$ par le cosinus de l'angle solaire zénithal $\theta_{\mathrm{S}}{ }^{t}$.

Puisque l'angle solaire zénithal dépend de la latitude, l'éclairement sur un plan horizontal dépend lui aussi de la latitude. Ceci est illustré par la figure 2 sur laquelle est représentée l'intégrale annuelle de l'éclairement, appelée irradiation* annuelle, au sommet de l'atmosphère pour un plan horizontal. On peut observer que cette irradiation ne dépend que de la latitude : l'irradiation est la même pour une latitude donnée, quelle que soit la longitude. Aux hautes latitudes, le Soleil est souvent bas sur l'horizon, même en plein été ; l'angle solaire zénithal est souvent grand et son cosinus souvent faible. Il s'ensuit que l'éclairement sur un plan horizontal est plus faible à ces hautes latitudes. Au contraire, autour de l'équateur, le Soleil sera haut dans le ciel tous les jours ; l'angle solaire zénithal prendra souvent des valeurs faibles et c'est là que sont observées les plus hautes valeurs d'éclairement au sommet de l'atmosphère. 


\section{Rayonnements direct, diffus et global}

La figure 3 schématise les effets de l'atmosphère sur les rayons descendants lors de leur traversée descendante vers la surface de la Terre. Même en l'absence de nuages, les rayons solaires sont partiellement absorbés par les gaz et diffusés par les molécules de l'air et les particules en suspension, solides ou liquides, appelés aérosols. La présence de nuages renforce les phénomènes de diffusion. L'absorption et la diffusion dépendent de la longueur d'onde du rayonnement incident. Les molécules de l'air diffusent davantage les petites longueurs d'onde (ultraviolet et bleu) que les grandes (rouge et infrarouge). C'est d'ailleurs pourquoi le ciel clair apparaît bleu hors la direction du Soleil. Sauf mention contraire, ce texte traite de l'éclairement total, c'est-à-dire de l'éclairement intégré sur tout le spectre solaire.

Bien que, au sommet de l'atmosphère, les rayons du Soleil ne proviennent que d'une direction, l'atmosphère agit comme un milieu diffusant et le rayonnement au sol provient de toutes les directions. De manière pratique, on distingue les composantes directes et diffuses de l'éclairement reçu au sol (figure 3).

La composante directe $B$ est l'éclairement provenant de la direction du Soleil et reçu sur un plan horizontal. C'est cette composante qui peut être concentrée dans les systèmes de conversion d'énergie dits à concentration puisque les rayons y sont parallèles, en première approximation. Les systèmes solaires à concentration, thermodynamiques ou photovoltaïques concentrés, seront de préférence installés dans les régions où cette composante est importante. Éclairement direct ne signifie pas que les photons n'ont pas été diffusés. Il s'agit de l'ensemble des photons, quel que soit le nombre de diffusions que chacun d'eux a subi, qui arrivent sur le plan horizontal en provenance de la direction du Soleil.

La composante diffuse $D$ est l'éclairement reçu sur le même plan horizontal depuis toutes les autres directions de la voûte céleste. Par ciel clair, c'est-àdire sans nuage, $B$ est plus élevé que $D$. L'éclairement global $G$ est la somme des rayonnements direct et diffus, $G=B+D$. Les systèmes photovoltaïques convertissent l'éclairement global et fonctionnent très bien même lorsque la composante diffuse domine.

\section{Rôle des aérosols et des nuages}

Les aérosols sont d'origine diverse : particules terrigènes ou écume de mer arrachées par les vents, pollens, émissions industrielles ou dues au trafic routier, marin et aérien, déforestation, pratiques agricoles et sylvicoles, éruptions volcaniques... Plus il y a d'aérosols dans la colonne atmosphérique, plus les photons sont absorbés mais surtout diffusés. La diffusion a lieu essentiellement vers l'avant,

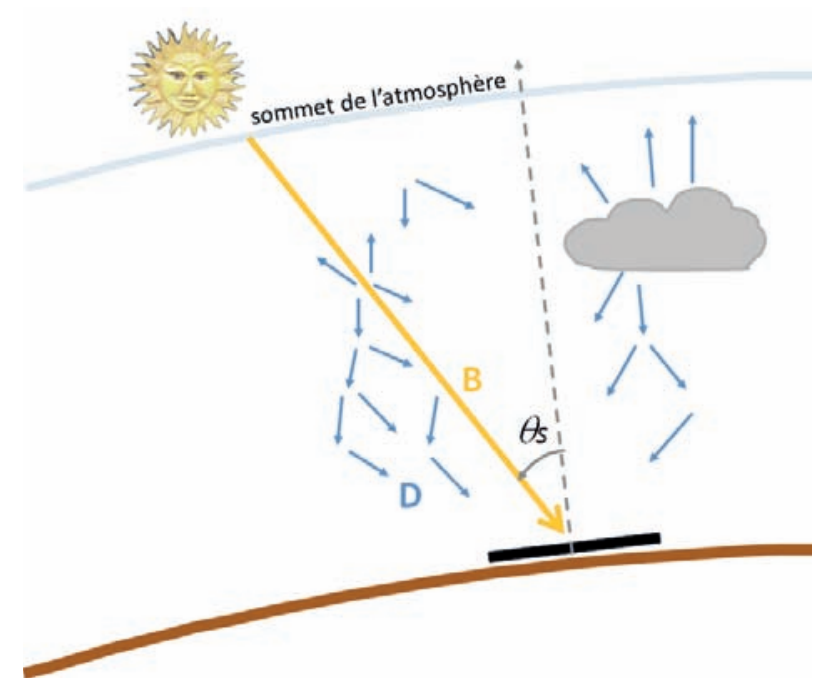

Figure 3. Les composantes directe $B$ et diffuse $D$ de l'éclairement global $G$ reçu au sol sur plan horizontal, $G=B+D$. $\theta_{S}$ est l'angle solaire zénithal.

c'est-à-dire dans un cône proche de la direction de propagation. Cependant, une partie des photons est diffusée dans d'autres directions ; par exemple, une partie est rétrodiffusée vers l'espace et n'atteint pas le sol. De manière générale, plus il y a d'aérosols, moins il y a de photons atteignant le sol.

Les aérosols ont un effet préjudiciable sur les systèmes solaires à concentration, car ils augmentent la composante diffuse de l'éclairement au détriment de la composante directe. Dans la péninsule arabique, par exemple, en l'absence de nuage, il est fréquent que la composante diffuse soit presqu'aussi grande que la composante directe à cause de l'abondance de poussières désertiques. Les systèmes photovoltaïques sont beaucoup moins sensibles à la présence d'aérosols, excepté pour les problèmes de salissure. Cette dernière va jouer le rôle d'un milieu diffusant et atténuer l'éclairement disponible pour la conversion photo-électrique.

Évidemment, les nuages jouent un rôle prépondérant sur l'éclairement au sol. Ils réfléchissent une partie des rayons descendants vers l'espace et diffusent le reste. De manière générale et excepté des cas particuliers de concentration des rayons solaires par une couverture nuageuse fragmentée, plus les nuages sont épais, plus ils réfléchissent et diffusent le rayonnement; par conséquent, moins il y a d'éclairement au sol. La part relative de la composante diffuse à l'éclairement global augmente rapidement avec l'épaisseur optique des nuages. Il suffit de nuages optiquement fins pour que la composante directe devienne nulle.

La figure 4 illustre la dépendance aux nuages de l'éclairement au sol. Elle représente l'irradiation annuelle au sol et est à rapprocher de l'irradiation annuelle au sommet de l'atmosphère présentée en figure 2. On observe que, comme au sommet de l'atmosphère, les minima sont situés aux hautes latitudes, les maxima sont plutôt dans les régions tropicales et équatoriales, et la distribution spatiale varie essentiellement avec la latitude. Cependant, on note de grandes différences entre les figures 2 et 4 . On observe par exemple un maximum sur l'Australie dont le couvert nuageux est assez faible et des irradiations relativement faibles dans les zones tropicales où persistent des nuages convectifs épais. Cette figure illustre la forte modulation par les nuages de l'éclairement au sol. 


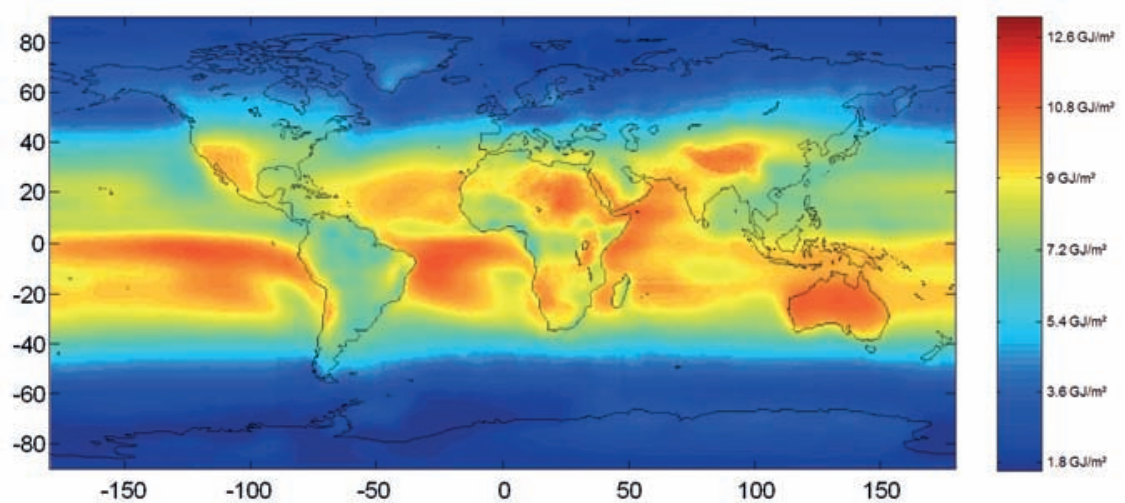

Figure 4. Irradiation annuelle au sol moyennée sur les années 1990-2004, en GJ m²- (c) Mines ParisTech 2006

\section{Comment estime-t-on l'éclairement?}

Les pyranomètres mesurent l'éclairement global et les pyrhéliomètres l'éclairement direct (figure 5). L'éclairement diffus est mesuré par un pyranomètre comprenant un élément mobile masquant la direction du Soleil.

Les pyranomètres et pyrhéliomètres constituent le meilleur moyen pour obtenir des mesures précises de l'éclairement. Cependant, il n'y en a pas assez pour couvrir la surface du globe et de nombreuses méthodes font appel aux images acquises par les satellites météorologiques pour obtenir une estimation de l'éclairement en tout point et à tout instant.

Les satellites météorologiques comme Meteosat Seconde Génération (MSG) produisent des images sur lesquelles on distingue bien les nuages (figure 6). Ces nuages sont généralement plus réfléchissants que les sols qu'ils couvrent. L'occurrence d'un nuage se traduit par une augmentation du signal perçu par le satellite par rapport à ce que ce même satellite devrait percevoir si le ciel était clair. Cette augmentation peut être liée à l'atténuation du rayonnement solaire

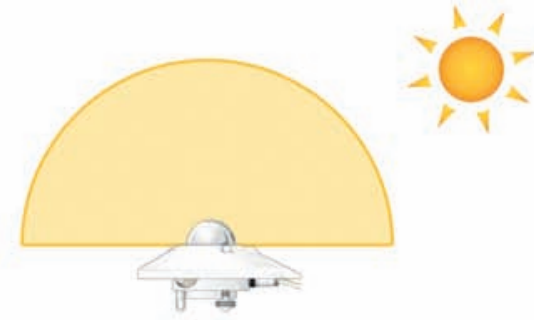

Pyranomètre : mesure de l'éclairement total global par intégration sur tout l'hémisphère depuis le sommet de l'atmosphère jusqu'au sol. Ce principe a été adopté

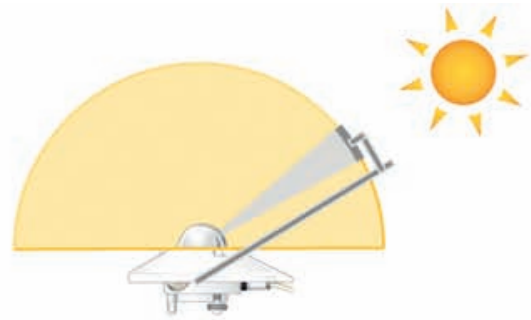

Pyranomètre ombré : mesure de l'éclairement total diffus par intégration de tout l'hémisphère à l'exception d'un angle solide autour du Soleil par de nombreuses méthodes pour l'estimation de l'éclairement. C'est le cas de la méthode Heliosat, sans doute la plus connue et la plus adoptée et adaptée, dont la première version a été proposée par Mines ParisTech en 1980, en collaboration avec M. Perrin de Brichambaut de Météo-France (Rigollier et al., 2004).

Depuis, les méthodes ont évolué vers une représentation de plus en plus précise des interactions entre le rayonnement et les constituants atmosphériques. Météo-France a notamment élaboré un modèle précis de détection de présence de nuages sur les images Meteosat et d'estimation de leurs propriétés optiques.

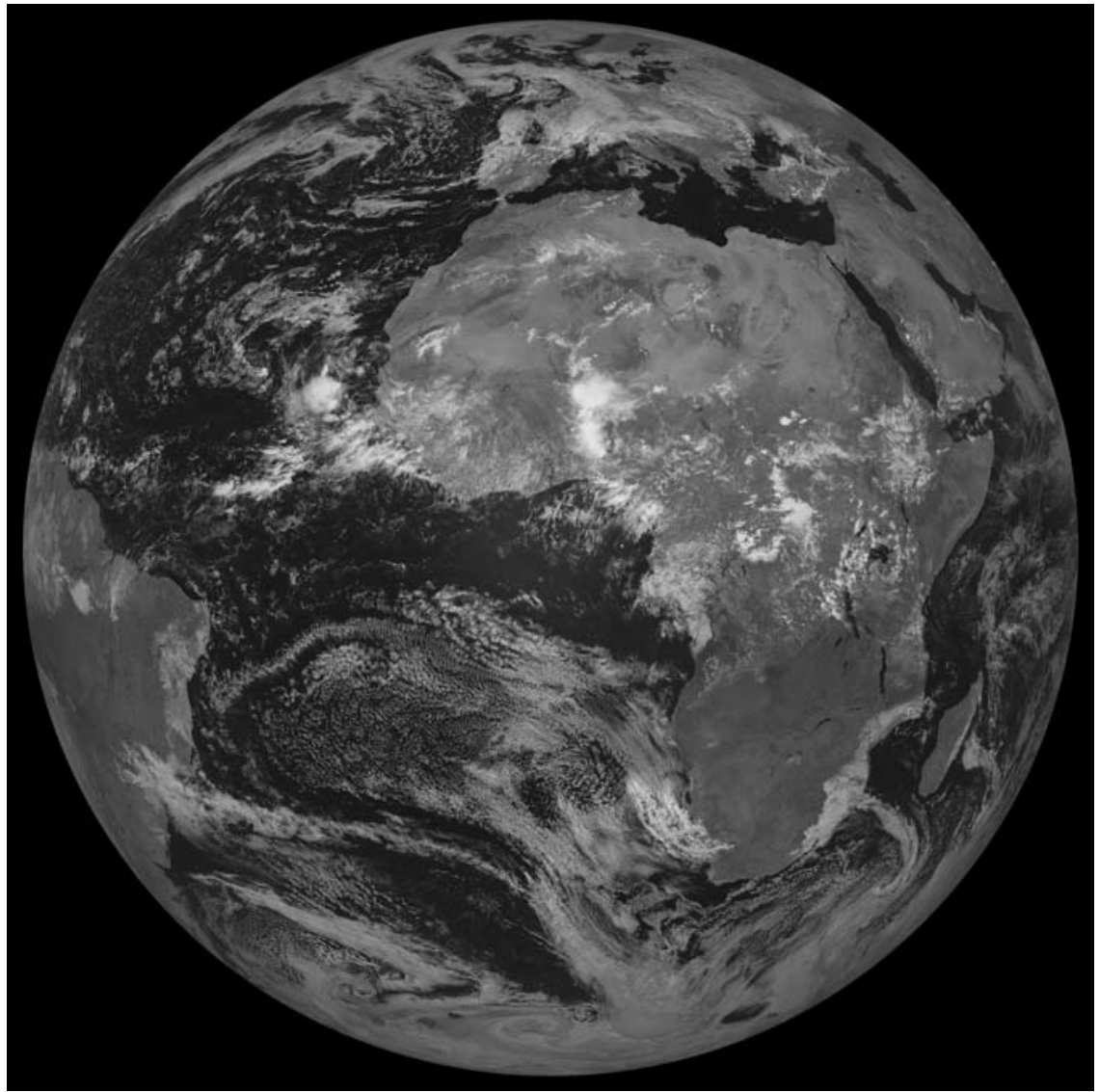

Figure 6. Exemple d'une image Meteosat-8, obtenue dans le canal visible le 7 septembre 2010 à $12 \mathrm{~h}$ UTC. La réflectance croît du noir au blanc. (c) Eumetsat (2010).

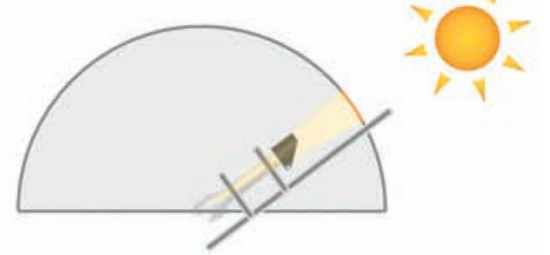

Pyrhéliomètre pointé vers le Soleil : mesure de l'éclairement total direct en incidence normale par intégration uniquement d'un angle solide autour du Soleil

Figure 5. Exemple de capteurs actinométriques par ordre croissant de complexité de l'instrumentation. 


\section{Genèse de la nouvelle méthode Heliosat-4}

Cependant, le point noir de ces méthodes restait l'estimation précise de l'éclairement par ciel clair, c'est-à-dire sans nuage. Lorsque le ciel est clair, les aérosols jouent un rôle prépondérant dans l'atténuation du rayonnement par leurs propriétés de diffusion et pour certains d'absorption. Les propriétés optiques des aérosols et leur concentration étaient peu connues il y a quelques années. Seules des cartes grossières de leur répartition, à large échelle spatiale et de type climatologique à l'échelle mensuelle ou annuelle, étaient proposées et utilisées par les méthodes d'estimation. Il s'ensuit que les éclairements par ciel clair estimées par ces méthodes utilisant des valeurs type pour les aérosols ne reproduisaient qu'imparfaitement les observations. L'éclairement par ciel clair représente la valeur la plus élevée de l'éclairement qui peut être observée, sauf cas particuliers. L'éclairement pour un ciel quelconque n'est qu'une fraction de cet éclairement de ciel clair. On conçoit donc l'importance d'une estimation précise dans le cas du ciel clair.

Heureusement, les projets MACC ont levé ce verrou en produisant et fournissant des estimations des concentrations en aérosols et de leurs propriétés optiques toutes les trois heures pour le monde entier. Mines ParisTech et l'agence aérospatiale allemande DLR (Deutsches Zentrums für Luft- und Raumfahrt) ont profité de ces nouveaux produits pour concevoir et réaliser la nouvelle méthode Heliosat-4 d'estimation de l'éclairement à partir des images Meteosat.

De nombreuses simulations numériques du transfert radiatif ont été effectuées durant les projets MACC afin d'estimer l'impact de la variation des constituants atmosphériques de ciel clair les plus notables - aérosols, ozone, vapeur d'eau - sur l'éclairement par ciel nuageux. Elles ont permis d'obtenir un résultat non intuitif concernant la séparation des effets du ciel clair sur l'éclairement par ciel nuageux, d'une grande portée pratique pour la nouvelle méthode Heliosat-4.

Désignons par $B, G, B_{\mathrm{c}}$ et $G_{\mathrm{c}}$ respectivement les éclairements direct et global sur plan horizontal pour tout ciel et par ciel clair (indice c). Les indices de ciel clair $K_{\mathrm{cb}}$ et $K_{\mathrm{cg}}$ sont définis par :
$B=B_{\mathrm{c}}\left(\theta_{\mathrm{S}}, P_{\mathrm{c}}\right) K_{\mathrm{cb}}\left(\theta_{\mathrm{S}}, P_{\mathrm{c}}, P_{\text {nuage }}\right)$
$G=G_{\mathrm{c}}\left(\theta_{\mathrm{S}}, \rho_{\mathrm{g}}, P_{\mathrm{c}}\right) K_{\mathrm{cg}}\left(\theta_{\mathrm{S}}, \rho_{\mathrm{g}}, P_{\mathrm{c}}, P_{\text {nuage }}\right)$

où $\theta_{\mathrm{S}}$ est l'angle solaire zénithal et $\rho_{\mathrm{g}}$ l'albédo du sol. $P_{\mathrm{c}}$ est un ensemble de variables décrivant 1'état optique de l'atmosphère claire : contenu de la colonne atmosphérique en ozone et vapeur d'eau, profil vertical de température, pression, densité et rapport de mélange des gaz, épaisseurs optiques des aérosols à 550 et $1100 \mathrm{~nm}$, type d'aérosol, altitude du sol au-dessus du niveau moyen de la mer. $P_{\text {nuage }}$ est un ensemble de variables décrivant le nuage : épaisseur optique, contenu en eau liquide ou en glace, rayon effectif des gouttelettes d'eau, phase du nuage (gouttelettes d'eau ou cristaux de glace), altitude de la base et du sommet.

Les indices $K_{\mathrm{cb}}$ et $K_{\mathrm{cg}}$ représentent l'influence des nuages sur l'éclairement. On s'attend à ce que ces indices varient avec les conditions $P_{\mathrm{c}}$ de ciel clair, puisque les nuages et les autres constituants sont mélangés dans 1'atmosphère. En fait, un des résultats de MACC est que les variations de $K_{\mathrm{cb}}$ et de $K_{\mathrm{cb}}$ avec $P_{\mathrm{c}}$ peuvent être négligées (Oumbe et al., 2014). L'équation (1) peut alors être remplacée par l'équation suivante avec une faible perte de précision :

$B \approx B_{\mathrm{c}}\left(\theta_{\mathrm{S}}, P_{\mathrm{c}}\right) K_{\mathrm{cb}}\left(\theta_{\mathrm{S}}, P_{\mathrm{c} 0}, P_{\text {nuage }}\right)$
$G \approx G_{\mathrm{c}}\left(\theta_{\mathrm{S}}, \rho_{\mathrm{g}}, P_{\mathrm{c}}\right) K_{\mathrm{cg}}\left(\theta_{\mathrm{S}}, \rho_{\mathrm{g}}, P_{\mathrm{c} 0}, P_{\text {nuage }}\right)$

où $P_{\mathrm{c} 0}$ est un ensemble type de variables décrivant l'état optique de l'atmosphère claire. L'erreur faite en utilisant cette approximation dépend essentiellement de l'angle solaire zénithal, l'albédo du sol et l'épaisseur optique du nuage. Dans la plupart des cas, l'erreur sur les éclairements direct et global, exprimée comme le $95^{\mathrm{e}}$ percentile, est inférieure à $15 \mathrm{~W} \mathrm{~m}^{-2}$, soit une erreur similaire aux recommandations de l'OMM pour des mesures de haute qualité $(3 \%)$.

Ce résultat a une grande portée pratique pour l'estimation de l'éclairement de manière opérationnelle. En effet, l'équation (2) signifie que deux modèles peuvent être développés séparément : un pour le cas du ciel clair $\left(B_{\mathrm{c}}\right.$ et $G_{\mathrm{c}}$ ), un autre pour les indices de ciel clair. En outre, la résolution de l'équation (1) nécessite un ré-échantillonnage spatial et temporel de chaque variable dans un référentiel commun, puisque $P_{\mathrm{c}}$ et $P_{\text {nuage }}$ doivent être connus aux mêmes instants et mêmes lieux. Au contraire, l'usage de l'équation (2) permet à chaque modèle d'avoir son propre jeu de données d'entrée avec ses propres résolutions temporelles et spatiales ; l'interpolation spatio-temporelle ne s'effectuera que sur $B_{\mathrm{c}}$ et $G_{\mathrm{c}}$ ou $K_{\mathrm{cb}}$ et $K_{\text {cg. }}$. En effet, le calcul des effets des nuages n'a besoin que d'un ensemble type $P_{\mathrm{c} 0}$ qui peut être représenté une fois pour toutes sur le même maillage que $P_{\text {nuage }}$

\section{Description de Heliosat-4}

La nouvelle méthode Heliosat-4 d'estimation de l'éclairement exploite le résultat précédent. Il aurait été possible d'utiliser des modèles existants, comme par exemple, le modèle ESRA (European Solar Radiation Atlas, Rigollier et al., 2000) ou le modèle Solis (Mueller et al., 2004) pour $B_{\mathrm{c}}$ et $G_{\text {c }}$. Deux nouveaux modèles ont été développés afin de profiter au mieux des estimations des constituants de l'atmosphère faites par les projets MACC. Le modèle McClear estime l'éclairement par ciel clair $B_{\mathrm{c}}$ et $G_{\mathrm{c}}$, et le modèle McCloud estime les indices de ciel clair $K_{\mathrm{cb}}$ et $K_{\mathrm{cg}}$. Les deux modèles sont détaillés ci-après.

La figure 7 présente le schéma de la méthode Heliosat-4. La méthode effectue d'abord les calculs du modèle McClear et, éventuellement, ceux du modèle McCloud, si un nuage est présent.

Les deux modèles McClear et McCloud ont été conçus en fonction des entrées disponibles opérationnellement. Ainsi, les variables de ciel clair $P_{\mathrm{c}}$ sont fournies par les projets MACC en ce qui concerne la composition de 1'atmosphère en gaz et aérosols. Le profil vertical de température, pression, densité et rapport de mélange des gaz provient d'une librairie de profils type AFGL (US Air Force Geophysics Laboratory). Le profil est choisi en fonction de la position géographique du lieu à partir d'une interprétation de la carte mondiale des climats de Trewartha (1954). L'altitude du sol est demandée à l'utilisateur. En cas d'absence de réponse, l'altitude est lue dans la base de données SRTM (Shuttle radar topography mission) de la Nasa et, pour les zones non couvertes par SRTM, dans la base Gtopo30 de l'USGS (US Geological Survey). L'albédo du sol est extrait d'un ensemble de cartes de moyennes mensuelles calculées par Mines ParisTech à partir de cartes hebdomadaires mises à la disposition de 


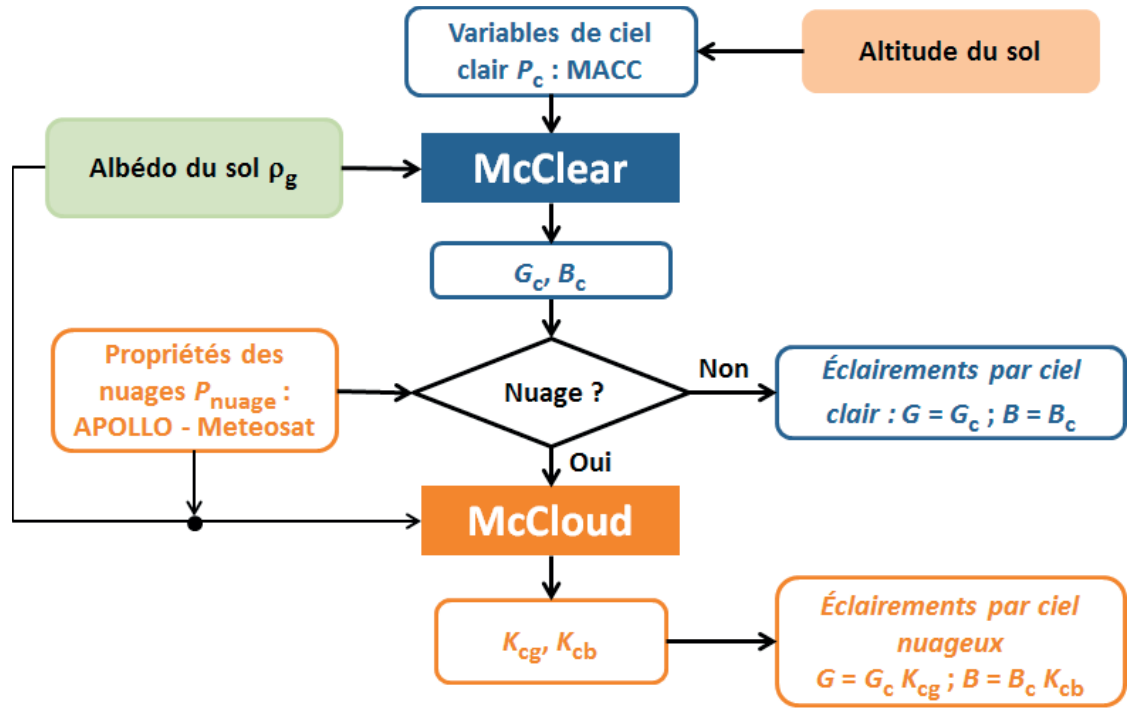

Figure 7. Schéma de la nouvelle méthode Heliosat-4.

chacun par la Nasa et estimées à partir d'observations du capteur spatioporté Modis (Moderate resolution imaging spectroradiometer). Toutes ces variables sont des entrées du modèle McClear, qui fournit en sortie les éclairements par ciel clair $B_{\mathrm{c}}$ et $G_{\mathrm{c}}$.

Le modèle McClear est en fait une approximation très rapide du modèle numérique libRadtran ${ }^{1}$ permettant de résoudre l'équation du transfert radiatif. Les modèles numériques de transfert radiatif reproduisent de manière précise les interactions entre le rayonnement et l'atmosphère, et permettent le calcul des éclairements au sol. Cependant, ils sont trop lents pour une utilisation opérationnelle. Pour pallier cette difficulté, McClear fait appel à des abaques. Un abaque est une table à multiples entrées donnant $B_{\mathrm{c}}$ et $G_{\mathrm{c}}$ pour des valeurs sélectionnées des entrées, que l'on appelle des nœuds. Les valeurs de rayonnement aux nœuds sont calculées une fois pour toutes grâce au modèle libRadtran, qui est exécuté pour chacun des jeux d'entrées sélectionnés. Ce calcul est très exigeant en termes de ressources informatiques, mais n'est effectué qu'une fois. Les éclairements sont ensuite calculés pour n'importe quel jeu de valeurs d'entrée par des interpolations des rayonnements connus aux nœuds.

S'il n'y a pas de nuage, la méthode Heliosat-4 s'achève ici, sachant que, dans ce cas, les éclairements $B$ et $G$ sont égaux à $B_{\mathrm{c}}$ et $G_{\mathrm{c}}$ (figure 7 ).

La présence ou non de nuage est décidée par une analyse des images Meteosat disponibles toutes les 15 minutes, selon

\footnotetext{
1. http://www.libradtran.org

2. AVHRR : Advanced Very High Resolution Radiometer.
}

la méthode Apollo (AVHRR 2 Processing Scheme Over Clouds, Land and Ocean), mise en œuvre par le DLR, qui exploite l'ensemble des données acquises dans les différents canaux spectraux de Meteosat. S'il y a un nuage au-dessus du lieu considéré à l'instant considéré, le modèle McCloud est exécuté. Ses entrées sont l'albédo du sol, les éclairements par ciel clair $B_{\mathrm{c}}$ et $G_{\mathrm{c}}$, et les propriétés des nuages $P_{\text {nuage }}$ (figure 7). La méthode Apollo estime également 1'épaisseur optique des nuages et leur type : nuage bas, nuage d'altitude moyenne, nuage haut et cirrus. Les trois premiers sont des nuages composés de gouttelettes d'eau, le quatrième de cristaux de glace. Ces variables constituent $P_{\text {nuage }}$.

Comme McClear, McCloud fait appel à des abaques, calculés à l'aide de libRadtran. Ces abaques diffèrent de ceux de McClear comme on peut le déduire de l'équation (2). Les entrées sont différentes, beaucoup moins nombreuses. Il en résulte des abaques moins volumineux à calculer, puis à charger en mémoire. McCloud calcule $K_{\text {cb }}$ et $K_{\text {cg }}$. La méthode Heliosat-4 effectue alors les produits de $B_{\mathrm{c}}$ par $K_{\mathrm{cb}}$ et de $G_{\mathrm{c}}$ par $K_{\mathrm{cg}}$ pour fournir $B$ et $G$ (figure 7).

Si le modèle McClear est applicable au monde entier, car ses entrées sont connues pour le monde entier, la méthode Apollo est restreinte au champ de vue du satellite Meteosat, ce qui limite la méthode Heliosat-4 à ce même champ de vue, soit environ un tiers du globe.

Les résultats d'Heliosat-4 ont été comparés avec des mesures d'éclairement de très haute qualité effectuées au pas temporel de 1 min, puis agrégées à $15 \mathrm{~min}$, dans quatorze stations du réseau BSRN (Baseline Surface Radiation Network). Pour l'éclairement global, on observe une surestimation. Le biais est la différence entre les moyennes des mesures et des estimations et quantifie cette surestimation. Il est compris entre 4 et $34 \mathrm{~W} \mathrm{~m}^{-2}$. La racine carrée de l'écart quadratique moyen donne une approximation de l'incertitude. Elle est d'environ $90 \mathrm{~W} \mathrm{~m}^{-2}$, soit entre 15 et $20 \%$ de l'éclairement mesuré dans les régions méditerranéennes et désertiques, et entre 26 et $45 \%$ pour les climats tempérés. Pour l'éclairement direct, le biais entre Heliosat-4 et les mesures est compris entre -44 et $46 \mathrm{~W} \mathrm{~m}^{-2}$. La racine carrée de l'écart quadratique moyen varie entre $81 \mathrm{~W} \mathrm{~m}^{-2}(32 \%$ de la moyenne de l'éclairement mesuré sur $15 \mathrm{~min})$ et $189 \mathrm{~W} \mathrm{~m}^{-2}(64 \%)$. En effectuant les comparaisons avec des irradiations horaires ou journalières, on obtient un résultat attendu, à savoir que le biais reste le même, car c'est une erreur systématique et une incertitude qui décroît. Heliosat-4 présente des biais et incertitudes similaires, voire plus faibles, que ceux des méthodes couramment utilisées, ce qui est satisfaisant pour une toute première version.

Si on se limite aux cas de ciel clair, c'est-à-dire sans nuage, les biais sont similaires au cas « tout ciel », mais les racines carrées des écarts quadratiques moyens sont nettement plus faibles : environ $35 \mathrm{~W} \mathrm{~m}^{-2}$ pour l'éclairement direct (5\% de l'éclairement mesuré) et entre $20 \mathrm{~W} \mathrm{~m}^{-2}$ (3\% de l'éclairement mesuré) et $64 \mathrm{~W} \mathrm{~m}^{-2}(10 \%)$ pour l'éclairement global (Lefèvre et al., 2013). Plus que la qualité du modèle lui-même, ces bonnes performances sont à mettre au crédit des estimations par MACC des propriétés des aérosols. Notons toutefois que les incertitudes sur ces propriétés sont encore grandes et que, par conséquent, les résultats de McClear n'atteignent pas la haute qualité requise par l'OMM. Des améliorations de l'estimation des propriétés des aérosols sont nécessaires pour accroître significativement la qualité des estimations de l'éclairement par ciel clair.

La grande différence entre les deux cas de ciel indique que des efforts sont à faire également sur la prise en compte des nuages. Des pistes d'amélioration ont été identifiées, portant essentiellement sur une meilleure détection des nuages, une meilleure estimation 
de leur épaisseur optique et une meilleure modélisation des nuages de type cumulonimbus de grande extension verticale.

\section{Accès aux estimations faites par Heliosat-4}

Mines ParisTech a fait le choix de ne pas créer de bases de données à proprement parler et de permettre à tout utilisateur d'exécuter Heliosat-4 à la volée. Ce choix pose de nombreux problèmes pratiques, mais l'un de ces avantages est de permettre des évolutions de Heliosat-4 sans recalculer une base de données.

Avant utilisation, un utilisateur doit s'enregistrer sur le site www.sodapro.com. Quelques minutes au plus sont nécessaires pour obtenir une série temporelle couvrant plusieurs années avec un pas temporel allant de $15 \mathrm{~min}$ à 1 journée. La figure 8 présente l'interface d'interrogation manuelle de Heliosat-4. Une application web, de type Web processing service, est également disponible pour permettre une automatisation des requêtes par les utilisateurs.

Depuis la mise en service de cette interface, soit en mars 2014, on compte 128 utilisateurs, dont environ la moitié de chercheurs, universitaires et étudiants, et la moitié d'entreprises. Le nombre élevé d'entreprises est surprenant, car il est rare que celles-ci bâtissent des procédures opérationnelles sur des systèmes balbutiants. Ceci témoigne certainement de la foi des entreprises d'une part dans la qualité des résultats des projets MACC et d'autre part dans la capacité du DLR et de Mines ParisTech à exploiter ces résultats pour l'estimation de l'éclairement.

Les estimations obtenues par satellite servent notamment à fabriquer des atlas de la ressource solaire, utilisés par les entreprises pour définir des régions favorables à l'implantation d'une centrale. Le site potentiel est ensuite sélectionné en prenant en compte d'autres variables de type géographique, accessibilité du site, possibilité de raccordement au réseau électrique, coût des terrains, soutiens politiques, acceptation par la population, etc. L'évaluation de la ressource solaire est un élément important. En effet, elle va permettre l'évaluation de la production d'électricité, donc celle

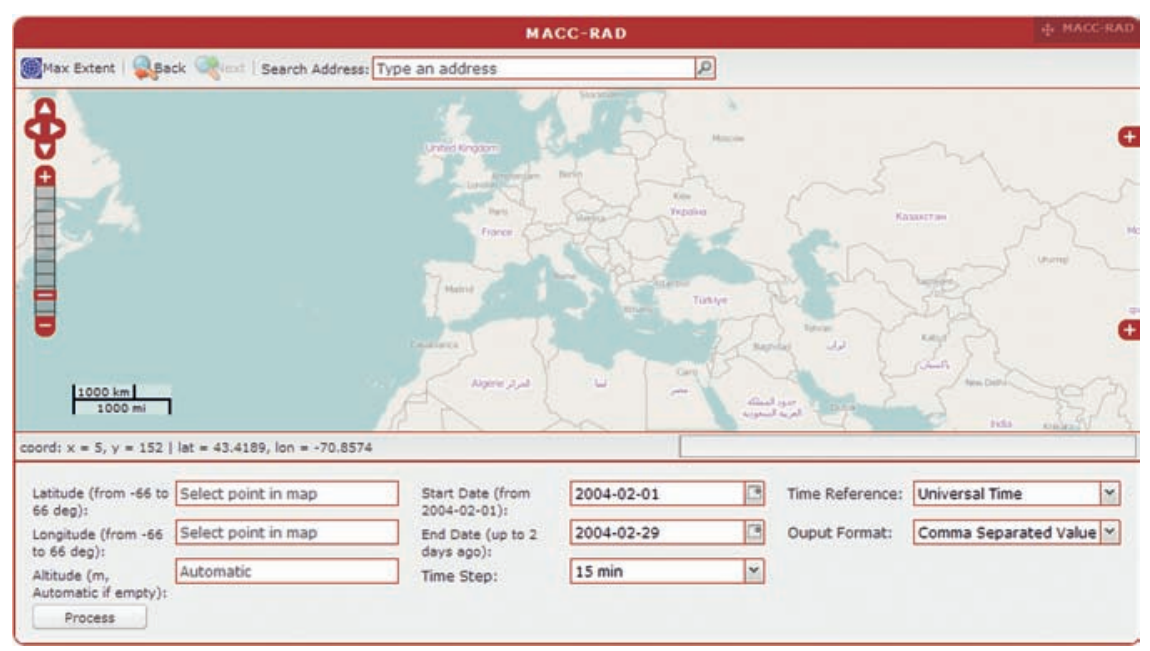

Figure 8. La société Transvalor développe le service SoDa (www.soda-pro.com), permettant l'accès aux estimations faites par Heliosat-4. $\mathrm{du}$ retour financier sur investissement et par conséquent l'acceptation du dossier par une banque.

\section{Exemple d'utilisation dans une demande d'investissement}

Acceptée par les banques, la pratique commune d'évaluation de la ressource est la suivante. Une station de mesure d'éclairement est installée sur le site pendant environ un an. Les mesures sont ensuite confrontées aux estimations coïncidentes effectuées par une méthode exploitant des images de satellite, Heliosat-4 par exemple, et les estimations sont alors ajustées aux mesures. Le modèle d'ajustement est étendu aux estimations Heliosat-4, mais pour une plus grande période, de 10 ans ou plus. Cette série ajustée d'estimations sert d'entrée à un simulateur numérique de la centrale solaire prévue afin d'obtenir la production potentielle.

Nous utilisons dans ce texte les mesures d'excellente qualité réalisées par MétéoFrance à Carpentras pour illustrer cet exemple d'application de Heliosat-4. Il s'agit d'un cas purement fictif et tout à fait particulier puisque les séries de mesures à Carpentras sont très longues et qu'il n'est nul besoin de les compléter par des estimations Heliosat-4 ou autres. Cependant, l'existence même de cette longue série permet dans cet exemple de justifier la pertinence de la pratique décrite ci-dessus. Nous nous limitons à

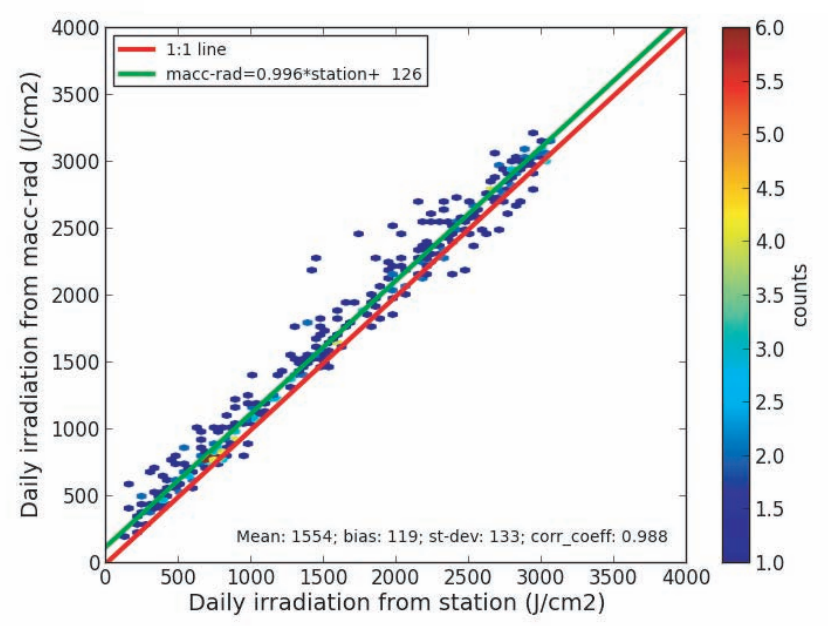

Figure 9. Histogramme bidimensionnel des mesures d'irradiation journalière à Carpentras en 2013 (axe horizontal) et des estimations effectuées par Heliosat-4 (axe vertical). L'échelle des couleurs à droite donne la relation entre la couleur de chaque point du graphe et le nombre d'occurrences de ce point. Dans le cas idéal, les points doivent être situés le long de la ligne rouge. II y a surestimation si les points sont situés au-dessus de la ligne rouge et sous-estimation s'ils sont situés en dessous. La ligne verte est la fonction affine correspondant au premier axe d'inertie de l'ensemble des points. Idéalement, elle devrait être confondue avec la ligne rouge. L'irradiation moyenne mesurée (" mean »), la moyenne (« bias »), l'écart type (« st-dev ») des erreurs et le coefficient de corrélation sont portés sur le graphe. 
l'irradiation globale jour-nalière au lieu d'irradiation mesurée toutes les $15 \mathrm{~min}$ ou $1 \mathrm{~h}$ pour des raisons d'illustration.

Conformément à la pratique, nous supposons qu'une station a été installée à Carpentras et qu'elle a collecté 1 an de mesures d'irradiation globale journalière faites durant 2013. La figure 9 présente les résultats de la confrontation entre les mesures et les estimations Heliosat-4 de l'irradiation journalière.

La figure 9 montre que les estimations Heliosat-4 reproduisent bien les mesures. La dispersion des points est faible, le coefficient de corrélation est très élevé $(0,988)$, la pente de la droite d'ajustement statistique (en vert) est quasiment égale à $1(0,996)$. Cependant, on note une surestimation de l'irradiation par
Heliosat-4 : presque tous les points sont au-dessus de la ligne rouge. Le biais est de $119 \mathrm{~J} \mathrm{~cm}^{-2}$, soit environ $8 \%$ de la moyenne d'irradiation mesurée.

Une fois inversée, la fonction affine déterminée sur la figure 9 permet de corriger les estimations Heliosat-4 en éliminant notamment le biais afin qu'elles se superposent au mieux aux mesures. Cet ajustement est étendu à l'ensemble des estimations Heliosat-4 pour la période 2004-2013. La figure 10 présente les résultats de la confrontation entre les mesures et les estimations, mais cette fois pour les 10 ans de données et avec les estimations Heliosat-4 ajustées.

On y voit que les estimations Heliosat-4 ajustées sont bien alignées avec les mesures coïncidentes. Le coefficient de corrélation est très élevé. L'écart type

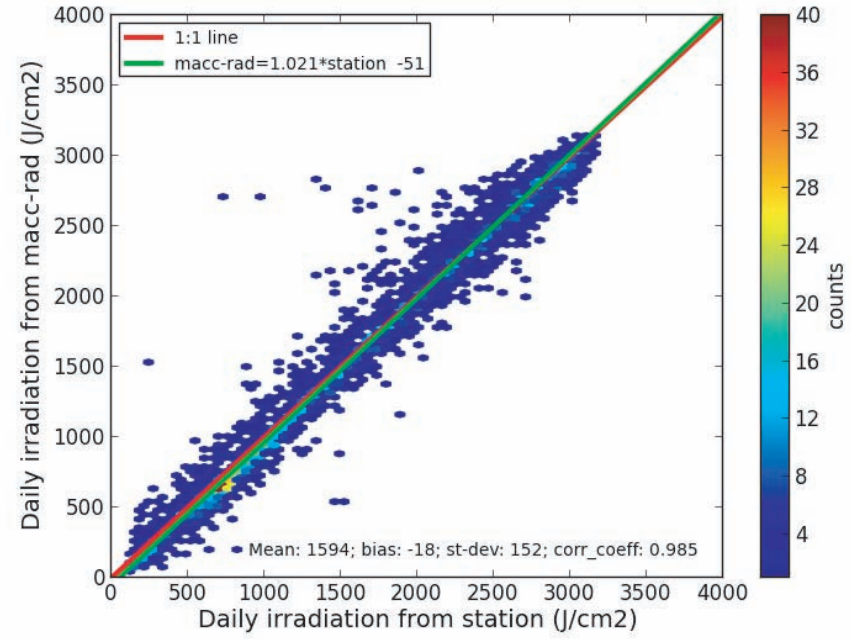

Figure 10. Même type de graphe que la figure 9, mais pour les mesures effectuées de 2004 à 2013 (axe horizontal) et les estimations Heliosat-4 ajustées (axe vertical).

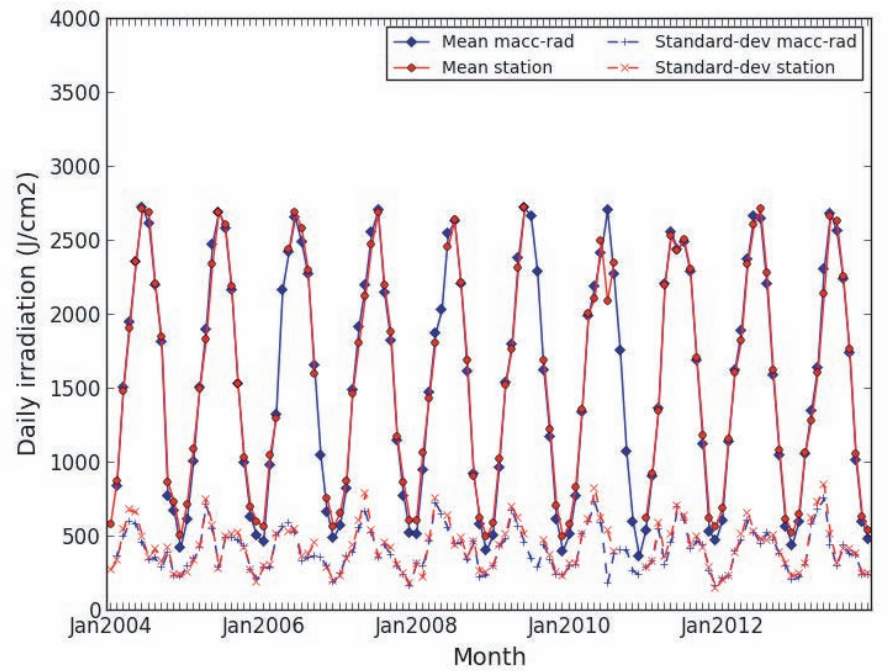

Figure 11. Moyennes mensuelles de l'irradiation journalière (cercles rouges pour les mesures de Météo-France et losanges bleus pour les estimations Heliosat-4 ajustées) et écarts types de cette irradiation au sein de chaque mois (respectivement croix rouges et croix bleues) pour la période de 10 ans. Le mois doit présenter au moins 25 valeurs journalières valides. des erreurs, qui caractérise la dispersion des points autour de la ligne idéale en rouge, est faible, inférieur à $10 \%$ de la valeur moyenne d'irradiation mesurée. Par rapport à la figure 9, on remarque que le biais a quasiment disparu. Il est passé de 119 à $18 \mathrm{~J} \mathrm{~cm}^{-2}$. On en déduit que l'ajustement calculé sur une année de mesures est parfaitement applicable à l'ensemble de la période. La figure 11 présente les moyennes mensuelles de l'irradiation journalière ainsi que les écarts types de cette irradiation au sein de chaque mois pendant la période de 10 ans, pour les mesures de Météo-France et les estimations Heliosat-4 ajustées.

À l'exception notable du mois de juillet 2010, les mesures et les estimations sont très proches les unes des autres, ce qui montre qu'une fois ajustées les estimations Heliosat-4 reproduisent bien les moyennes mensuelles de l'irradiation journalière et la variation de cette dernière dans un mois, exprimée par l'écart type. L'industriel peut alors entrer cette série Heliosat- 4 ajustée dans son simulateur numérique de la centrale solaire. Cet exemple montre que Heliosat- 4 se prête bien à cet usage par les professionnels en énergie solaire, qui en fait appliquent cette pratique aux irradiations horaires ou intégrées sur $15 \mathrm{~min}$.

\section{Conclusion}

En proposant de nouvelles données sur les concentrations en aérosols et leurs propriétés optiques, sur les contenus en vapeur d'eau et ozone, et sur les propriétés optiques des nuages, les projets MACC ont permis la réalisation d'une nouvelle méthode opérationnelle, Heliosat-4, fondée sur une résolution directe de l'équation du transfert radiatif. Quoique dans sa toute première version, cette méthode fournit des estimations de l'éclairement global et de ses composantes directe et diffuse reçus sur un plan horizontal au sol de qualité similaire à celles des méthodes actuelles.

Le concept innovant de Heliosat-4 repose sur deux résultats importants des projets MACC en ce qui concerne la modélisation du transfert radiatif. Le premier résultat est que les variations des indices de ciel clair avec la composition de l'atmosphère claire peuvent être négligées. De manière pratique, cela permet de développer deux modèles séparément, l'un pour l'éclairement par ciel clair, l'autre pour les indices de ciel clair, c'est-à-dire l'atténuation engendrée par la présence de nuages. $\mathrm{Ce}$ 
résultat dépasse largement le cadre de MACC et de Heliosat-4.

Le deuxième résultat est le modèle de ciel clair McClear qui se révèle être d'une grande précision grâce aux nouvelles données fournies par MACC, bien que des améliorations soient nécessaires. Ce modèle a été implanté dans d'autres instituts que Mines ParisTech. Il est également utilisé en conjonction avec la base de données HelioClim-3, produite par Transvalor et très utilisée par les professionnels, et en diminue l'incertitude (Qu et al., 2014).
Le travail sur Heliosat-4, McCloud et McClear devrait continuer pendant plusieurs années. Plusieurs améliorations sont déjà en cours de réalisation ou de validation. Elles portent sur l'estimation des propriétés optiques, d'une part des aérosols, d'autre part des nuages.

Du point de vue de la recherche, Heliosat-4, par sa prise en compte de la physique du transfert radiatif, ouvre de plus la voie vers une caractérisation plus fine de l'éclairement au sol comme sa répartition spectrale ou encore sa répartition angulaire.

\section{Remerciements}

Les auteurs remercient Olivier Boucher et Benoît Gschwind pour leurs relectures de ce texte et leurs suggestions. La méthode Heliosat- 4 résulte de recherches partiellement financées par l'Union européenne : convention de recherche FP $7 n^{\circ} 218793$ (projet MACC), FP 7 $\mathrm{n}^{\circ} 283576$ (projet MACC-II) et H2020 $\mathrm{n}^{\circ} 633080$ (projet MACC-III).

\section{Bibliographie}

Kopp G., Lean J.L., 2011. A new, lower value of total solar irradiance: evidence and climate significance. Geophys. Res. Lett., 38, L01706. doi: 10.1029/2010GL045777 Lefèvre M., Oumbe A., Blanc P., Espinar B., Gschwind B., Qu Z., Wald L., Schroedter-Homscheidt M., Hoyer-Klick C., Arola A., Benedetti A., Kaiser J.W., Morcrette J.-J., 2013. McClear: a new model estimating downwelling solar radiation at ground level in clear-sky conditions. Atmos. Meas. Tech., 6, 2403-2418. doi: 10.5194/amt-6-24032013

Meftah M., Dewitte S., Irbah A., Chevalier A., Conscience C., Crommelinck D., Janssen E., Mekaoui S., 2004. SOVAP/Picard, a spaceborne radiometer to measure the total solar irradiance. Sol. Phys. , 289, 1885-1899. doi: 10.1007/s11207-013-0443-0

Mueller R.W., Dagestad K.F., Ineichen P., Schroedter M., Cros S., Dumortier D., Kuhlemann R., Olseth J.A., Piernavieja G., Reise C., Wald L., Heinnemann D., 2004. Rethinking satellite based solar irradiance modelling - The SOLIS clear sky module. Remote Sens. Environ., 91, 160-174. doi: 10.1016/.j.re.2004.02.009

Oumbe A., Qu Z., Blanc P., Lefèvre M., Wald L., Cros S., 2014. Decoupling the effects of clear atmosphere and clouds to simplify calculations of the broadband solar irradiance at ground level. Geosci. Model Dev., 7, 1661-1669. doi: 10.5194/gmd-7-1661-2014

Qu Z., Gschwind B., Lefèvre M., Wald L., 2014. Improving HelioClim-3 estimates of surface solar irradiance using the McClear clear-sky model and recent advances in atmosphere composition. Atmos. Meas. Tech., 7, 3927-3933. doi: 10.5194/amt-7-3927-2014

Rigollier C., Bauer 0., Wald L., 2000. On the clear sky model of the ESRA - European Solar Radiation Atlas - with respect to the Heliosat method. Sol. Energy, 68, 33-48. doi: 10.1016/S0038-092X(99)00055-9

Rigollier C., Lefèvre M., Wald L., 2004. The method Heliosat-2 for deriving shortwave solar radiation from satellite images. Sol. Energy, 77(2), 159-169. doi: 10.1016/j.solener.2004.04.017

Trewartha G.T., 1954. An Introduction to Climate. 3rd Edn, McGraw Hill Book Co.
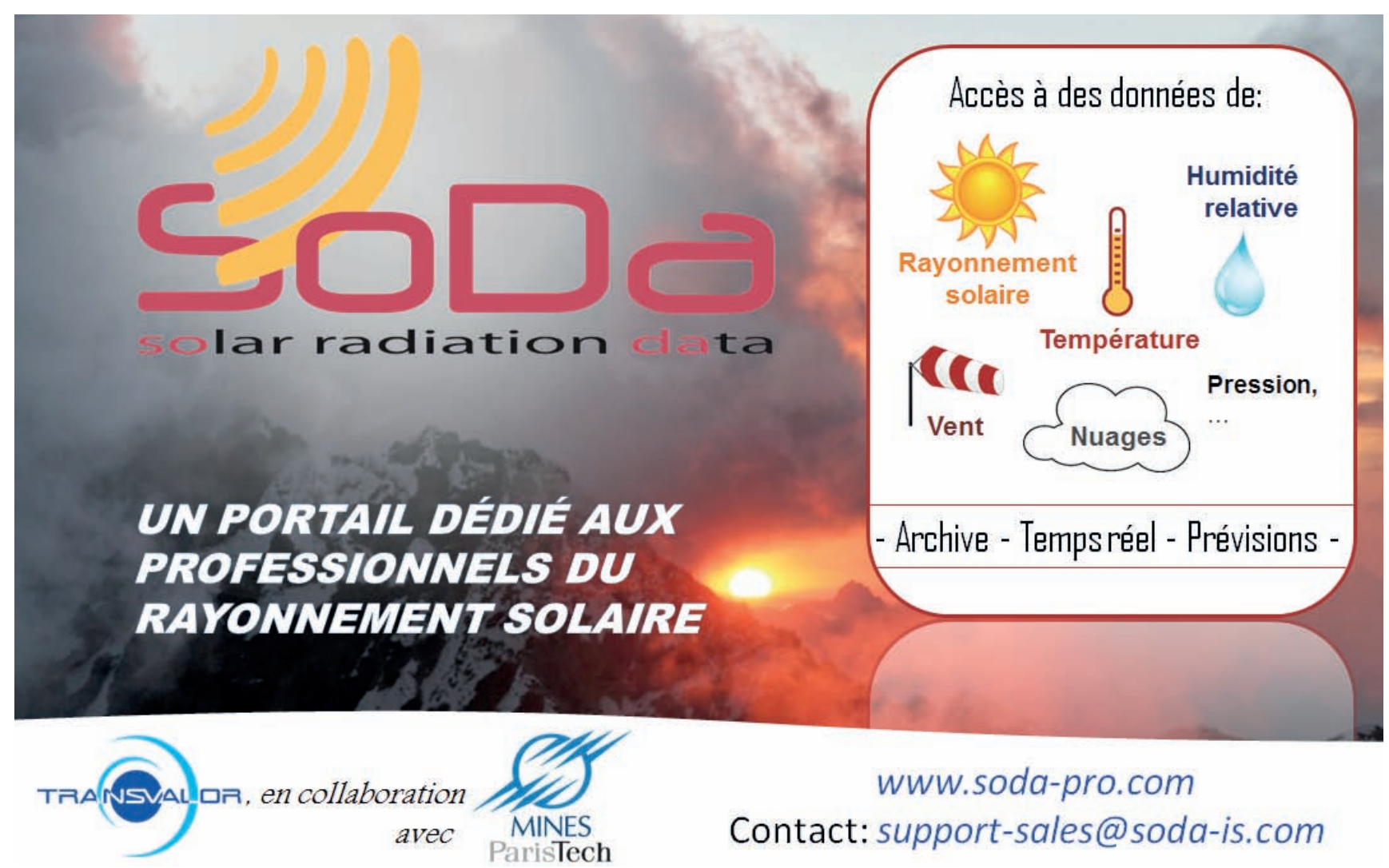